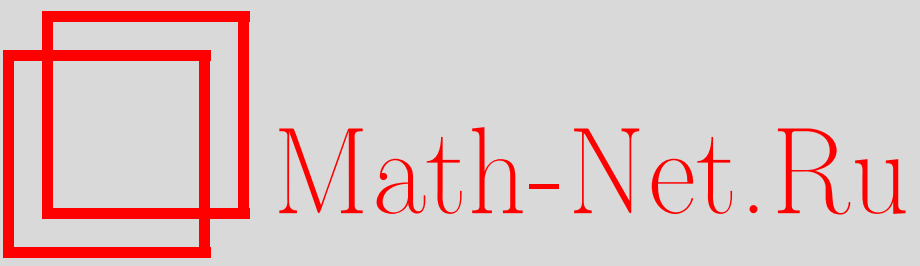

Л. В. Богданов, Е. В. Ферапонтов, Нелокальный гамильтонов формализм полугамильтоновых систем гидродинамического типа, ТМФ, 1998, том 116, номер 1, 113-121

DOI: https://doi.org/10.4213/tmf891

Использование Общероссийского математического портала Math-Net.Ru подразумевает, что вы прочитали и согласны с пользовательским соглашением

http: //www . mathnet.ru/rus/agreement

Параметры загрузки:

IP : 35.173 .219 .149

26 апреля 2023 г., 16:32:13 
ТЕОРЕТИЧЕСКАЯ

И МАТЕМАТИЧЕСКАЯ

ФИЗИКА

Том 116, № 1

июль, 1998

(C) 1998 г.

Л.В. Богданов* , Е. В. Ферапонтов*

\section{НЕЛОКАЛЬНЫЙ ГАМИЛЬТОНОВ ФОРМАЛИЗМ ПОЛУГАМИЛЬТОНОВЫХ СИСТЕМ ГИДРОДИНАМИЧЕСКОГО ТИПА}

С использованием формальных функций Бейкера-Ахиезера для $(2+1)$-мерной системы $n$ волн строится нелокальный гамильтонов формализм полугамильтоновых систем гидродинамического типа.

\section{1. ВВЕДЕНИЕ}

Согласно результатам работы [1] система гидродинамического типа в инвариантах Римана, удовлетворяющая уравнениям

$$
R_{t}^{i}=v^{i}(R) R_{x}^{i}, \quad i=1, \ldots, n,
$$

является гамильтоновой с невырожденной скобкой Дубровина-Новикова тогда и только тогда, когда сушествует плоская диагональная метрика $\sum g_{i i}\left(d R^{i}\right)^{2}$ такая, что

$$
\partial_{j} \ln \sqrt{g_{i i}}=\frac{\partial_{j} v^{i}}{v^{j}-v^{i}}, \quad i \neq j
$$

где $\partial_{j}=\partial / \partial R^{j}$. В этом случае уравнения (1) представимы в гамильтоновой форме

$$
R_{t}^{i}=A^{i j} \frac{\delta H}{\delta R^{j}}
$$

с гамильтоновым оператором типа оператора Дубровина-Новикова [2]

$$
A^{i j}=\delta^{i j} g^{i i} d-g^{i i} \Gamma_{i k}^{j} R_{x}^{k}, \quad d=\frac{d}{d x}
$$

(здесь $g^{i i}=1 / g_{i i}, \Gamma_{i k}^{j}-$ символы Кристоффеля связности Леви-Чивита, порожденной метрикой $d s^{2}$ ) и подходящим гамильтонианом $H=\int h(R) d x$. Перекрестное дифференцирование соотношения (2) приводит к равенствам

$$
\partial_{k} \frac{\partial_{j} v^{i}}{v^{j}-v^{i}}=\partial_{j} \frac{\partial_{k} v^{i}}{v^{k}-v^{i}}, \quad i \neq j \neq k \neq i,
$$

\footnotetext{
${ }^{*}$ Институт теоретической физики им. Л.Д. Ландау, Черноголовка, Московская обл., Россия
} 
которые тем самым выполнены для любой гамильтоновой системы (1). Системы (1), характеристические скорости которых удовлетворяют соотношениям (5), были названы полугамильтоновыми. Поскольку общее решение уравнений (5) зависит от $n(n-1)$ функций двух аргументов (в то время как гамильтоновы системы (1), как и плоские диагональные метрики, параметризуются $n(n-1) / 2$ функциями двух аргументов), класс полугамильтоновых систем оказывается сушественно шире класса гамильтоновых. Всякая полугамильтонова система обладает бесконечным набором коммутирующих потоков

$$
R_{\tau}^{i}=w^{i}(R) R_{x}^{i}
$$

характеристические скорости которых удовлетворяют линейной системе уравнений

$$
\frac{\partial_{j} w^{i}}{w^{j}-w^{i}}=\frac{\partial_{j} v^{i}}{v^{j}-v^{i}}, \quad i \neq j .
$$

В работах $[3,4]$ было высказано предположение, согласно которому полугамильтоновы системы также являются гамильтоновыми, если от локальных гамильтоновых операторов (4) перейти к рассмотрению нелокальных (т.е. явно зависящих от $d^{-1}$ ) выражений вида

$$
A^{i j}=\delta^{i j} g^{i i} d-g^{i i} \Gamma_{i k}^{j} R_{x}^{k}+\sum_{\alpha} \varepsilon_{\alpha} w_{\alpha}^{i} R_{x}^{i} d^{-1} w_{\alpha}^{j} R_{x}^{j}, \quad \varepsilon_{\alpha}= \pm 1 .
$$

Гамильтоновы операторы (7) подробно рассматривались в работах $[3,4]$ как естественное обобшение нелокального гамильтонова формализма, предложенного в [5]. Процедура введения нелокального гамильтонова формализма выглядит следующим образом.

1. Для заданной полугамильтоновой системы (1) определяются коэффициенты метрики $d s^{2}=\sum g_{i i}\left(d R^{i}\right)^{2}$ из уравнений $(2)$. Заметим, что эти уравнения задают $g_{i i}$ с точностью до преобразований вида $g_{i i} \rightarrow g_{i i} / \varphi^{i}\left(R^{i}\right)$, где $\varphi^{i}$ является произвольной функцией аргумента $R^{i}$. Можно показать, что условия полугамильтоновости равносильны обрашению в нуль компонент $R_{k k j}^{i}$ тензора кривизны метрики $d s^{2}$ при $i \neq j \neq k \neq i$.

2. Ищется разложение оставшихся компонент $R_{i j}^{i j}=g^{i i} R_{i i j}^{j}$ тензора кривизны по квадратам решений линейной системы (6):

$$
R_{i j}^{i j}=\sum_{\alpha} \varepsilon_{\alpha} w_{\alpha}^{i} w_{\alpha}^{j}, \quad \varepsilon_{\alpha}= \pm 1 .
$$

Предположение о сушествовании разложений вида (8) высказывалось в работах $[3,4]$ и подтверждается многочисленными примерами. Строгое обоснование формулы (8) и является предметом данной работы. Заметим, что в (8) фактически приходится рассматривать бесконечные суммы $\sum_{\alpha}$ или даже интегралы $\int d \alpha$.

3. Как только разложение (8) найдено, система (1) может быть представлена в гамильтоновой форме (3) с нелокальным гамильтоновым оператором (7). При этом тождества Якоби для оператора (7) сводятся к соотношениям (2), (6), (8). Поскольку метрика $d s^{2}$ определяется с произволом в $n$ функций одного аргумента $\varphi^{i}\left(R^{i}\right)$, всякая полугамильтонова система допускает бесконечное число согласованных нелокальных гамильтоновых структур.

Таким образом, построение нелокального гамильтонова формализма сводится к обоснованию разложений вида (8). 
ПРИмЕР 1. Рассмотрим двухкомпонентную слабонелинейную систему

$$
R_{t}^{1}=R^{2} R_{x}^{1}, \quad R_{t}^{2}=R_{x}^{2} R^{1}
$$

Соответствуюшие уравнения (2) принимают форму

$$
\partial_{2} \ln \sqrt{g_{11}}=\frac{1}{R^{1}-R^{2}}, \quad \partial_{1} \ln \sqrt{g_{22}}=\frac{1}{R^{2}-R^{1}},
$$

так что

$$
g_{11}=\frac{1}{\left(R^{1}-R^{2}\right)^{2} \varphi^{1}\left(R^{1}\right)}, \quad g_{22}=\frac{1}{\left(R^{1}-R^{2}\right)^{2} \varphi^{2}\left(R^{2}\right)},
$$

где $\varphi^{i}\left(R^{i}\right)$ - две произвольные функции одной переменной. Для системы (8) линейная система (6) может быть явно проинтегрирована:

$$
w^{1}=\frac{1}{2} \varphi^{1^{\prime}}\left(R^{1}-R^{2}\right)-\frac{1}{2}\left(\varphi^{1}+\varphi^{2}\right), \quad w^{2}=\frac{1}{2} \varphi^{2^{\prime}}\left(R^{2}-R^{1}\right)-\frac{1}{2}\left(\varphi^{1}+\varphi^{2}\right) .
$$

Прямое вычисление показывает, что при данном выборе коэффициентов метрики $g_{11}$ и $g_{22}$ и коммутирующих потоков $w^{1}, w^{2}$ единственная ненулевая компонента $R_{12}^{12}$ тензора кривизны может быть представлена в виде

$$
R_{12}^{12}=w^{1}+w^{2}
$$

Так как

$$
w^{1}+w^{2}=\left(w^{1}+\frac{1}{2}\right)\left(w^{2}+\frac{1}{2}\right)-\left(w^{1}-\frac{1}{2}\right)\left(w^{2}-\frac{1}{2}\right),
$$

это разложение имеет искомый вид (8). Оно порождает нелокальный оператор

$$
A^{i j}=g^{i i} \delta^{i j} d-g^{i i} \Gamma_{i k}^{j} u_{x}^{k}+w^{i} R_{x}^{i} d^{-1} R_{x}^{j}+R_{x}^{i} d^{-1} w^{j} R_{x}^{j},
$$

который в матричной форме может быть представлен следуюшим образом:

$$
\begin{aligned}
A= & \left(R^{1}-R^{2}\right)^{2}\left(\begin{array}{cc}
\varphi^{1} & 0 \\
0 & \varphi^{2}
\end{array}\right) d+ \\
& +\frac{1}{2}\left(\begin{array}{cc}
\varphi^{1}\left(R^{1}-R^{2}\right)^{2} R_{x}^{1}+\varphi^{1}\left(R^{1}-R^{2}\right)_{x}^{2} & 2\left(R^{1}-R^{2}\right)\left(\varphi^{2} R_{x}^{1}+\varphi^{1} R_{x}^{2}\right) \\
2\left(R^{2}-R^{1}\right)\left(\varphi^{2} R_{x}^{1}+\varphi^{1} R_{x}^{2}\right) & \varphi^{2^{\prime}}\left(R^{1}-R^{2}\right)^{2} R_{x}^{2}+\varphi^{2}\left(R^{1}-R^{2}\right)_{x}^{2}
\end{array}\right)+ \\
& +\left(\begin{array}{cc}
w^{1} R_{x}^{1} d^{-1} R_{x}^{1}+R_{x}^{1} d^{-1} w^{1} R_{x}^{1} & w^{1} R_{x}^{1} d^{-1} R_{x}^{2}+R_{x}^{1} d^{-1} w^{2} R_{x}^{2} \\
w^{2} R_{x}^{2} d^{-1} R_{x}^{1}+R_{x}^{2} d^{-1} w^{1} R_{x}^{1} & w^{2} R_{x}^{2} d^{-1} R_{x}^{2}+R_{x}^{2} d^{-1} w^{2} R_{x}^{2}
\end{array}\right) .
\end{aligned}
$$

Таким образом, изучаемая система имеет бесконечно много нелокальных гамильтоновых структур с конечными нелокальными хвостами. 
Пример $2[3,4]$. Система

$$
R_{t}^{i}=\left(\sum R^{k}+2 R^{i}\right) R_{x}^{i}
$$

рассматривалась в статье [6] как бездисперсионный предел связанных уравнений Кортевега-де Фриза. Соответствуюшие уравнения (2) принимают форму

$$
\partial_{j} \ln \sqrt{g_{i i}}=\frac{1}{2\left(R^{j}-R^{i}\right)}, \quad i \neq j .
$$

Следовательно, получаем

$$
g_{i i}=\frac{\prod_{k \neq i}\left(R^{k}-R^{i}\right)}{\varphi^{i}\left(R^{i}\right)},
$$

где $\varphi^{i}\left(R^{i}\right)-n$ произвольных функций одной переменной. Выбирая $\varphi^{i}\left(R^{i}\right)=\left(R^{i}\right)^{a}$, $a=0, \ldots, n$, получаем $(n+1)$ плоских метрик, порождающих локальные гамильтоновы структуры типа структур Дубровина-Новикова. Оказывается, что в случае произвольных $\varphi^{i}$ метрика $g_{i i}$ также порождает нелокальный гамильтонов оператор, но с бесконечным нелокальным “хвостом”. Действительно, система (6) имеет частные решения вида

$$
w^{i}=\left(\left(R^{i}-\alpha\right) \sqrt{\prod_{k=1}^{n}\left(R^{k}-\alpha\right)}\right)^{-1}
$$

где $\alpha=$ const. Прямое вычисление вычетов показывает, что тензор кривизны $R_{i j}^{i j}$ метрики $g_{i i}$ может быть представлен в виде

$$
R_{i j}^{i j}=\frac{1}{8 \pi i} \int_{\gamma_{1}} w^{i} w^{j} \varphi^{1}(\alpha) d \alpha+\cdots+\frac{1}{8 \pi i} \int_{\gamma_{n}} w^{i} w^{j} \varphi^{n}(\alpha) d \alpha,
$$

где $\gamma_{i}$ - контур в комплексной $\alpha$-плоскости, окружающий точку $\alpha=R^{i}$. Нелокальный "хвост", соответствуюший этому разложению, имеет вид

$$
\frac{1}{8 \pi i} \int_{\gamma_{1}} w^{i} R_{x}^{i} d^{-1} w^{j} R_{x}^{j} \varphi^{1}(\alpha) d \alpha+\cdots+\frac{1}{8 \pi i} \int_{\gamma_{n}} w^{i} R_{x}^{i} d^{-1} w^{j} R_{x}^{j} \varphi^{n}(\alpha) d \alpha
$$

\section{2. ПЕРЕФОРМУЛИРОВКА ЗАДАЧИ НА ЯЗЫКЕ}

\section{КОЭФФИЦИЕНТОВ ЛАМЕ И КОЭФФИЦИЕНТОВ ВРАЩЕНИЯ}

Преобразуем разложение (8) к эквивалентной форме, введя коэффициенты Ламе $H^{i}=\sqrt{g_{i i}}$ и коэффициенты врашения $\beta_{i j}$ по формулам

$$
\partial_{i} H^{j}=\beta_{i j} H^{i}
$$

Тогда, как несложно проверить,

$$
R_{i j}^{i j}=\frac{1}{H^{i} H^{j}}\left(\partial_{i} \beta_{i j}+\partial_{j} \beta_{j i}+\sum_{k \neq i, j} \beta_{k i} \beta_{k j}\right), \quad w_{\alpha}^{i}=\frac{H_{\alpha}^{i}}{H^{i}}
$$


где $H_{\alpha}^{i}$ удовлетворяют [7] линейной системе (9), вследствие чего разложение (8) принимает вид

$$
\partial_{i} \beta_{i j}+\partial_{j} \beta_{j i}+\sum_{k \neq i, j} \beta_{k i} \beta_{k j}=\sum_{\alpha} \varepsilon_{\alpha} H_{\alpha}^{i} H_{\alpha}^{j}
$$

Формулы (9) представляют собой линейную задачу для системы $n$ волн

$$
\partial_{k} \beta_{i j}=\beta_{i k} \beta_{k j}
$$

Отметим, что уравнения (11) эквивалентны условиям полугамильтоновости (5). Ниже мы обоснуем разложение (10) для широкого класса решений системы (11), которые могут быть получены в рамках теории солитонов. Методы теории солитонов позволяют строить решения системы (11) вместе с волновыми функциями соответствуюших линейных уравнений. Далее мы приведем соответствующее построение в рамках метода $\bar{\partial}$-одевания [8].

\section{3. ФОРМАЛЬНЫЕ ФУНКЦИИ БЕЙКЕРА-АХИЕЗЕРА}

В этом разделе мы определим формальную матричную функцию Бейкера-Ахиезера как специальньй набор компонент волновых функций линейной задачи (9), зависящих от дополнительного, спектрального, параметра, и докажем, что разложение типа (10) имеет место для произвольных коэффициентов врашения, для которых существует матричная функция Бейкера-Ахиезера, определенная в виде формального ряда Лорана.

Для уравнений (9) при $i \neq j$ определим $n$ наборов формальных решений $\vec{H}_{(i)}=$ $\left(H_{(i)}^{1}, \ldots, H_{(i)}^{n}\right)^{t}$, заданных своими разложениями в окрестности бесконечности по комплексной переменной $\alpha$ :

$$
\vec{H}_{(i)}(\alpha)=\left(\begin{array}{c}
\beta_{i 1} \alpha^{-1}+q_{i 1} \alpha^{-2}+\cdots \\
\ldots \ldots \ldots \ldots \ldots \ldots \ldots \ldots \\
1+\beta_{i i} \alpha^{-1}+q_{i i} \alpha^{-2}+\cdots \\
\ldots \ldots \ldots \ldots \ldots \ldots \ldots \ldots \\
\beta_{i n} \alpha^{-1}+q_{i n} \alpha^{-2}+\cdots
\end{array}\right) \exp \left(\alpha R^{i}\right)
$$

где многоточием обозначены члены порядка $O\left(\alpha^{-3}\right)$. Компоненты $H_{(i)}^{k}(\alpha)$ образуют матричную функцию Бейкера-Ахиезера. Далее мы предполагаем, что матричная функция Бейкера-Ахиезера сушествует в виде формального ряда Лорана

$$
H_{(k)}^{i}(\alpha)=\sum_{n=-\infty}^{n=\infty} \psi_{i k}(n) \alpha^{n-1} .
$$

Коэффициенты ряда Лорана также являются волновыми функциями уравнений (9), т.к. эти уравнения не содержат $\alpha$.

В рамках метода $\bar{\partial}$-одевания эта функция конструируется как решение матричной нелокальной $\bar{\partial}$-проблемы на комплексной плоскости переменной $\alpha$ с подходящим одеванием ядра (одновременно строятся и соответствующие коэффищиенты вращения). 
Подстановка (12) в (9) приводит к следуюшему набору соотношений между коэффициентами $\beta_{i j}, \beta_{i i}$ и $q_{i j}$ :

$$
\begin{aligned}
\partial_{j} \beta_{i i} & =\beta_{i j} \beta_{j i}, & & i \neq j, \\
q_{i j} & =\beta_{i i} \beta_{i j}-\partial_{i} \beta_{i j}, & & i \neq j, \\
\partial_{k} \beta_{i j} & =\beta_{i k} \beta_{k j}, & & i \neq j \neq k .
\end{aligned}
$$

Дальнейшее использование подстановки (12) в (9) приводит к набору рекуррентных соотношений, позволяюших построить формальную функцию Бейкера-Ахиезера по коэффициентам врашения $\beta_{i j}$ в виде произведения экспоненциального множителя на формальный ряд по $\alpha$ (с точностью до обрашения операторов $\partial_{i}$ ). Таким образом, формальная функция Бейкера-Ахиезера может быть построена без использования конструктивных методов теории солитонов непосредственно по произвольным коэффициентам врашения. Однако коэффициенты ряда Лорана для формальной функции Бейкера-Ахиезера содержат бесконечные суммирования и могут расходиться.

Разложение вектор-функции $\vec{H}_{(i)}(-\alpha)$ на бесконечности имеет вид

$$
\vec{H}_{(i)}(-\alpha)=\left(\begin{array}{c}
-\beta_{i 1} \alpha^{-1}+q_{i 1} \alpha^{-2}+\cdots \\
\ldots \ldots \ldots \ldots \ldots \ldots \\
1-\beta_{i i} \alpha^{-1}+q_{i i} \alpha^{-2}+\cdots \\
\ldots \ldots \ldots \ldots \ldots \\
-\beta_{i n} \alpha^{-1}+q_{i n} \alpha^{-2}+\cdots
\end{array}\right) \exp \left(-\alpha R^{i}\right)
$$

Рассмотрим функцию

$$
H_{(k)}^{i}(\alpha) H_{(k)}^{j}(-\alpha)+H_{(k)}^{j}(\alpha) H_{(k)}^{i}(-\alpha),
$$

составленную из компонент вектор-функций $\vec{H}_{(k)}(\alpha)$ и $\vec{H}_{(k)}(-\alpha)$. Пользуясь формулами $(12),(14)$, мы находим разложение (15) в окрестности бесконечности:

$$
H_{(k)}^{i}(\alpha) H_{(k)}^{j}(-\alpha)+H_{(k)}^{j}(\alpha) H_{(k)}^{i}(-\alpha)= \begin{cases}-2 \partial_{i} \beta_{i j} \alpha^{-2}+\cdots & \text { при } k=i, \\ -2 \partial_{j} \beta_{j i} \alpha^{-2}+\cdots & \text { при } k=j, \\ -2 \beta_{k i} \beta_{k j} \alpha^{-2}+\cdots & \text { при } k \neq i, j,\end{cases}
$$

где многоточием обозначены члены порядка $O\left(\alpha^{-3}\right)$. Следовательно, имеем

$$
\begin{aligned}
\partial_{i} \beta_{i j} & +\partial_{j} \beta_{j i}+\sum_{k \neq i, j} \beta_{k i} \beta_{k j}= \\
= & \frac{1}{2} \sum_{k=1}^{n} \operatorname{Res}_{\infty} \alpha\left(H_{(k)}^{i}(\alpha) H_{(k)}^{j}(-\alpha)+H_{(k)}^{j}(\alpha) H_{(k)}^{i}(-\alpha)\right)= \\
= & \frac{1}{2} \sum_{k=1}^{n} \sum_{p=-\infty}^{p=\infty}(-1)^{p}\left(\psi_{i k}(p) \psi_{j k}(-p)+\psi_{j k}(p) \psi_{i k}(-p)\right)
\end{aligned}
$$


или, если ряд Лорана определяет функцию комплексной переменной $\alpha$ в некоторой окрестности $G$ бесконечности,

$$
\begin{aligned}
\partial_{i} \beta_{i j} & +\partial_{j} \beta_{j i}+\sum_{k \neq i, j} \beta_{k i} \beta_{k j}= \\
& =\frac{1}{4 \pi i} \sum_{k=1}^{n} \int_{\gamma} \alpha d \alpha\left(H_{(k)}^{i}(\alpha) H_{(k)}^{j}(-\alpha)+H_{(k)}^{j}(\alpha) H_{(k)}^{i}(-\alpha)\right),
\end{aligned}
$$

где $\gamma$ обозначает контур в окрестности бесконечности (границу $G)$. Формула $\left(16^{\prime}\right)$ тесно связана с известным результатом об интегрируемости уравнений, описывающих $n$-ортогональные криволинейные системы координат [9]. В силу элементарного тождества

$$
\begin{aligned}
H_{(k)}^{i}(\alpha) & H_{(k)}^{j}(-\alpha)+H_{(k)}^{j}(\alpha) H_{(k)}^{i}(-\alpha)= \\
= & \left(H_{(k)}^{i}(\alpha)+H_{(k)}^{i}(-\alpha)\right)\left(H_{(k)}^{j}(\alpha)+H_{(k)}^{j}(-\alpha)\right)- \\
& -H_{(k)}^{i}(-\alpha) H_{(k)}^{j}(-\alpha)-H_{(k)}^{i}(\alpha) H_{(k)}^{j}(\alpha)
\end{aligned}
$$

разложения $(16),\left(16^{\prime}\right)$ имеют искомый вид (8) (в разложении $\left(16^{\prime}\right)$ волновые фунции являются комплексными, поэтому необходимо переписать разложение в терминах вешественных и мнимых частей волновых функций, что не изменит искомую структуру разложения).

\section{4. ПОСТРОЕНИЕ ФУНКЦИЙ БЕЙКЕРА-АХИЕЗЕРА: МЕТОД}

Мы приведем краткое изложение хорошо известной схемы метода $\bar{\partial}$-одевания для уравнений (9), (11) (см. [8]) и обсудим особенности разложения (10) для точно решаемых случаев.

Введем нелокальную матричную $\bar{\partial}$-проблему с канонической нормировкой

$$
\begin{aligned}
\bar{\partial}_{\alpha} \chi(\alpha, R)= & \iint_{\mathbf{C}} d \alpha^{\prime} \wedge d \bar{\alpha}^{\prime} \chi\left(\alpha^{\prime}, R\right) \times \\
& \times \exp \left(\alpha^{\prime} \sum_{k=1}^{n} P^{(k)} R^{k}\right) K\left(\alpha, \alpha^{\prime}\right) \exp \left(-\alpha \sum_{k=1}^{n} P^{(k)} R^{k}\right), \\
& (\chi(\alpha, R)-1)_{|\alpha| \rightarrow \infty} \rightarrow 0,
\end{aligned}
$$

где $\chi(\alpha, R)$ и ядро $K\left(\alpha, \alpha^{\prime}\right)$ - матричнозначные $n \times n$-функции, $P^{(k)}$ - диагональные матрицы с единственным ненулевым элементом (единицей) на месте $k, P_{i j}^{(k)}=\delta_{i k} \delta_{j k}$. Задача (17) сводится к интегральному уравнению.

Мы предполагаем, что ядро $K\left(\alpha, \alpha^{\prime}\right)$ равно нулю в некоторой окрестности бесконечности $G$ по переменным $\alpha$ и $\alpha^{\prime}$, тогда функция $\chi(\alpha, R)$ аналитична в этой окрестности.

В терминах функции Бейкера-Ахиезера

$$
\psi(\alpha, R)=\chi(\alpha, R) \exp \left(\alpha \sum_{k=1}^{n} P^{(k)} R^{k}\right)
$$


задача (17) принимает вид

$$
\bar{\partial}_{\alpha} \psi(\alpha, R)=\iint_{\mathbf{C}} d \alpha^{\prime} \wedge d \bar{\alpha}^{\prime} \psi\left(\alpha^{\prime}, R\right) K\left(\alpha, \alpha^{\prime}\right) .
$$

В соответствии с методом $\bar{\partial}$-одевания предположение об однозначной разрешимости задачи (17) приводит к линейным уранениям на функцию $\chi$

$$
P^{(j)}\left(\partial_{i} \chi(\alpha, R)+\alpha \chi(\alpha, R) P^{(i)}\right)=P^{(j)} U(R) P^{(i)} \chi(\alpha, R)
$$

или в терминах функции $\psi-$

$$
P^{(j)} \partial_{i} \psi(\alpha, R)=P^{(j)} U(R) P^{(i)} \psi(\alpha, R),
$$

где функция $U(R)$ определяется разложением $\chi(\alpha, R)$ по $\alpha$ на бесконечности:

$$
\chi(\alpha, R) \rightarrow 1+\frac{U(R)}{\alpha}+\cdots, \quad \alpha \rightarrow \infty
$$

Записывая матричное уравнение (20) в компонентах, получаем

$$
\partial_{i} \psi_{j k}(\alpha, R)=U_{j i}(R) \psi_{i k}(\alpha, R)
$$

Это уравнение при сопоставлении $H_{(k)}^{i}=\psi_{i k}, \beta_{i j}=U_{j i}$ совпадает с уравнением (9) для компонент матричной функции Бейкера-Ахиезера. Разложение (12) следует из определения функции $\psi$. Таким образом, задача (17) позволяет строить решения уравнений (10) и матричные функции Бейкера-Ахиезера (12), зависящие от функционального параметра - ядра $K\left(\alpha, \alpha^{\prime}\right)$.

В случае вырожденных ядер

$$
K\left(\alpha, \alpha^{\prime}\right)=\sum_{k=1}^{P} f^{(k)}\left(\alpha^{\prime}\right) g^{(k)}(\alpha)
$$

включающем решения типа солитонных, задача (17) сводится к системе линейных уравнений и решается явно. Разложение $\left(16^{\prime}\right)$ в этом случае сильно упрощается. Действительно, перейдем в правой части $\left(16^{\prime}\right)$ от интегрирования по контуру к интегрированию по области, дополнительной к $G$ (для простоты мы выпишем только один член), и воспользуемся уравнением (18):

$$
\begin{aligned}
& \int_{\gamma}\left(H_{(k)}^{i}(\alpha) H_{(k)}^{j}(-\alpha)\right) \alpha d \alpha= \\
& =\iint_{\mathbf{C} \backslash \bar{G}} \alpha d \alpha \wedge d \bar{\alpha} \bar{\partial}_{\alpha}\left(H_{(k)}^{i}(\alpha) H_{(k)}^{j}(-\alpha)\right)= \\
& =\sum_{p=1}^{n} \iint_{\mathbf{C}} \alpha d \alpha \wedge d \bar{\alpha} \iint_{\mathbf{C}} d \alpha^{\prime} \wedge d \bar{\alpha}^{\prime}\left(H_{(p)}^{i}\left(\alpha^{\prime}, R\right) K_{p k}\left(\alpha, \alpha^{\prime}\right) H_{(k)}^{j}(-\alpha)+\right. \\
& \left.\quad+H_{(p)}^{j}\left(\alpha^{\prime}, R\right) K_{p k}\left(\alpha, \alpha^{\prime}\right) H_{(k)}^{i}(-\alpha)\right) .
\end{aligned}
$$


В случае вырожденных ядер получаем, что

$$
\begin{aligned}
\sum_{p=1}^{n} \iint_{\mathbf{C}} \alpha d \alpha \wedge d \bar{\alpha} \iint_{\mathbf{C}} d \alpha^{\prime} \wedge d \bar{\alpha}^{\prime}\left(H_{(p)}^{j}\left(\alpha^{\prime}, R\right) K_{p k}\left(\alpha, \alpha^{\prime}\right) H_{(k)}^{j}(-\alpha)+\right. \\
\left.\quad+H_{(p)}^{j}\left(\alpha^{\prime}, R\right) K_{p k}\left(\alpha, \alpha^{\prime}\right) H_{(k)}^{i}(-\alpha)\right)= \\
=\sum_{r=1}^{P} \sum_{q=1}^{n}\left\{\iint_{\mathbf{C}} \alpha d \alpha \wedge d \bar{\alpha} g_{q k}^{(r)}(\alpha) H_{(k)}^{j}(-\alpha) \times\right. \\
\quad \times \sum_{p=1}^{n} \iint_{\mathbf{C}} d \alpha^{\prime} \wedge d \bar{\alpha}^{\prime} H_{(p)}^{i}\left(\alpha^{\prime}, R\right) f_{p q}^{(r)}\left(\alpha^{\prime}\right)+ \\
\quad+\iint_{\mathbf{C}} \alpha d \alpha \wedge d \bar{\alpha} g_{q k}^{(r)}(\alpha) H_{(k)}^{i}(-\alpha) \times \\
\left.\quad \times \sum_{p=1}^{n} \iint_{\mathbf{C}} d \alpha^{\prime} \wedge d \bar{\alpha}^{\prime} H_{(p)}^{j}\left(\alpha^{\prime}, R\right) f_{p q}^{(r)}\left(\alpha^{\prime}\right)\right\}= \\
=\sum_{r=1}^{P} \sum_{q=1}^{n}\left(\widetilde{H}_{r q}^{\prime i} \times \widetilde{H}_{r q}^{\prime \prime} j+\widetilde{H}_{r q}^{\prime j} \times \widetilde{H}_{r q}^{\prime \prime}\right),
\end{aligned}
$$

где $\widetilde{H}_{r q}^{\prime i}, \widetilde{H}_{r q}^{\prime \prime} i$ представляют собой два различных набора волновых фукций уравнений (9). Используя элементарное тождество, которое применялось для разложения (16), приходим к выводу, что в случае вырожденных ядер имеется разложение типа (10) в виде конечной суммы.

\section{Список литературы}

[1] С. П. Царев. ДАН СССР. 1985. Т. 282. № 5. С. 534-537.

[2] Б. А. Дубровин, С. П. Новиков. ДАН СССР. 1983. Т. 270. № 4. С. 781-785.

[3] Е. В. Ферапонтов. Функц. анализ и его прилож. 1991. Т. 25. Вып. 3. С. 37-49.

[4] E. V. Ferapontov. Amer. Math. Soc. Transl. (2). 1995. V. 170. P. 33-58.

[5] О.И. Мохов, Е. В. Ферапонтов. УМН. 1990. Т. 45. Вып. 3. С. 191-192.

[6] E. V. Ferapontov, M. V. Pavlov. Physica D. 1991. V. 52. P. 211-219.

[7] С. П. царев. Изв. АН СССР, сер. матем. 1990. Т. 54. № 5. С. 1048-1068.

[8] С. В. Манаков, В. Е. Захаров. Функц. анализ и его прилож. 1985. Т. 19. Вып. 2. С. 11-25.

[9] V.E. Zakharov. Description of the $n$-orthogonal curvilinear coordinate systems and Hamiltonian Integrable Systems of Hydrodynamic Type: Part 1. Integration of the Lamé equations. Preprint ITEP, 1997. P. 1-42. 